$\alpha$-dystroglycan to the extracellular matrix in the muscles of normal, healthy mice, without introducing any abnormalities.

The researchers then decided to test the effects of LARGE in the cells of patients with CMD. They treated the cells of patients with three different types of CMD - Fukuyama CMD, muscle-eye-brain disease and Walker-Warburg syndrome - with the adenovirus that carried the LARGE gene. And although these diseases are caused by mutations in different glycosyltransferase enzymes, expression of LARGE generated functional, glycosylated $\alpha$-dystroglycan in all three cases.

These results, say the authors, suggest that stimulating the addition of sugar to $\alpha$-dystroglycan by LARGE could provide a potential therapy for certain muscular dystrophies, regardless of the type of glycosyltransferase enzyme that has been mutated.

Clare Ellis

(Q) References and links ORIGINAL RESEARCH PAPERS Barresi, R. et al. LARGE can functionally bypass $\alpha$-dystroglycan glycosylation defects in distinct congenital muscular dystrophies. Nature Med. 6 June 2004 (doi:10.1038/nm1059) | Kanagawa, M. et al. Molecular recognition by LARGE is essential for expression of functional dystroglycan. Cell 3 June 2004 (doi:101016/S0092867404005434)

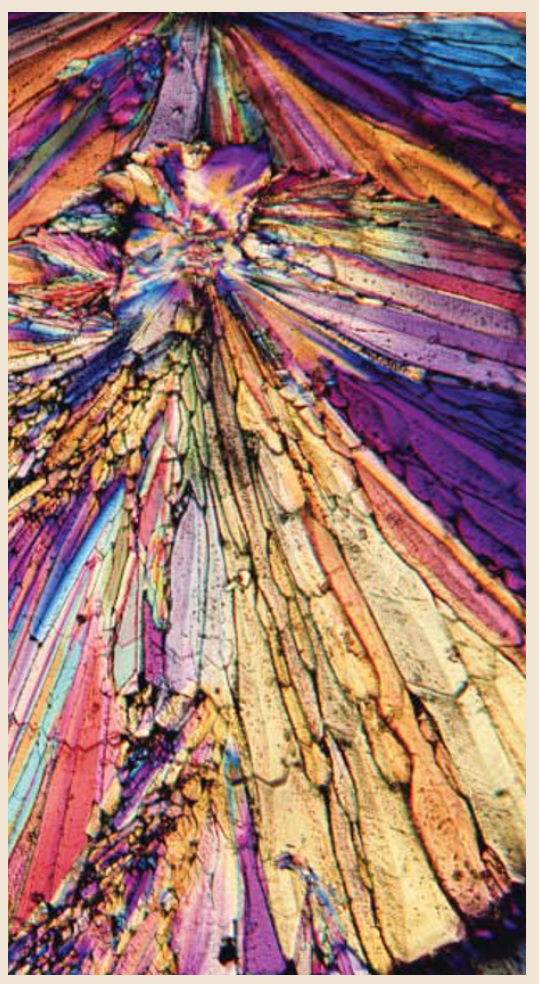

\section{HIGH-THROUGHPUT SCREENING}

\section{Free your assay}

Many strategies for high-throughput screening (HTS) against enzyme targets require the analytes to be labelled, which has a number of potential disadvantages, such as interference of the label with the enzyme activity being assessed. Mass spectrometry (MS) methods do not have this problem, but the need for several time-consuming steps in analyte preparation has limited the use of such methods in HTS. Writing in Nature

Biotechnology, Milan Mrksich and colleagues now describe a new MS-based assay strategy in which analyte preparation is sufficiently simple to allow rapid screening of chemical libraries, which they demonstrate by identifying a potent inhibitor of the anthrax lethal factor.

At the core of the authors' strategy are specially designed self-assembled monolayers (SAMs), which present enzyme substrates on a gold layer in a way that ensures that interactions with soluble enzymes only occur by way of the immobilized substrates. Then, after one washing step, these layers are suitable for analysis using matrix-assisted laser-desorption ionization time-of-flight (MALDI-TOF) MS, allowing any change in the mass of the substrate owing to an enzymatic reaction to be readily detected.

To identify inhibitors of anthrax lethal factor, a protease with a key role in anthrax pathogenesis, Mrksich et al. produced $10 \times 10$ arrays of reaction wells lined with SAMs that displayed a peptide substrate for lethal factor. Analysis of the SAMs by MALDI-TOF MS (a procedure that the authors termed SAMDI) before and after treatment with lethal factor clearly showed a change in mass of the surface-linked peptide resulting from the loss of an $\mathrm{N}$-terminal fragment by proteolytic cleavage.

Then, to screen a 10,000-compound library against lethal factor, the authors incubated cocktails containing lethal factor and 8 library compounds in each well on the $10 \times 10$ arrays. SAMDI analysis indicated that complete cleavage of the peptide occurred in most wells, but 11 of the wells $(\sim 1 \%)$ showed no or only partial proteolysis of the peptide, indicating the presence of an inhibitor in the cocktail. Repeating the assay with the 88 compounds present in these cocktails identified one compound, DS-998, that completely blocked lethal factor activity at $10 \mu \mathrm{m}$ concentration. This low-molecular-mass compound was also shown to be active in cell-based assays, and so represents a potential lead for anti-anthrax drug discovery efforts.

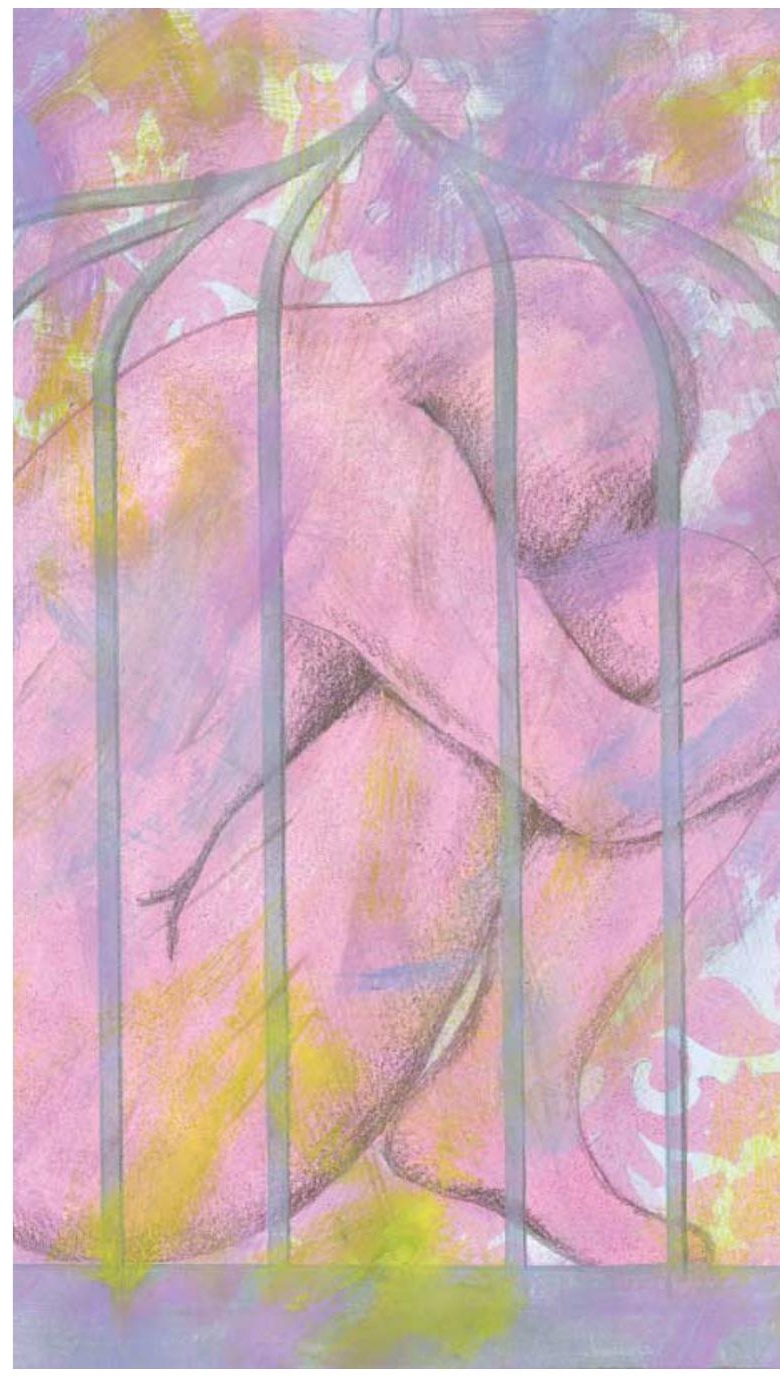

So, how does the robustness of the SAMDI method, in terms of distinguishing positive signals from background, compare with others used in chemical screening? A widely accepted parameter for assay quality is the $Z^{\prime}$ factor, which ranges from 1 for a 'perfect' assay to 0 . The authors determined the $Z^{\prime}$ factor of their SAMDI assay to be 0.83 , which compares very favourably with established assays. It therefore seems that the SAMDI method has significant potential as a rapid and general label-free screening approach for enzyme targets, and it could be especially valuable for assays involving enzymes that are not easily monitored with labels, such as carbohydrate-modifying enzymes.

Peter Kirkpatrick

\section{(2) References and links}

ORIGINAL RESEARCH PAPER Min, D.-H., Teng, W.-J. \& Mrksich, M. Chemical screening by mass spectrometry to identify inhibitors of anthrax lethal factor. Nature Biotechnol. 22, 717-723 (2004)

FURTHER READING Su, J. \& Mrksich, M. Using mass spectrometry to characterize self-assembled monolayers presenting peptides, proteins, and carbohydrates. Angew. Chem. Intl Edn $\mathbf{4 1}$, (c) 2004 Nature Publishing G715-478(2002) 